\title{
Behaviour of Al2O3 in aluminium matrix composites: An overview
}

\author{
Lokesh Singh $^{1}$, Shankar Sehgal ${ }^{2, *}$, and Kuldeep K. Saxena ${ }^{1}$ \\ ${ }^{1}$ Department of Mechanical Engineering, GLA University, Mathura, 281406, India \\ ${ }^{2}$ Mechanical Engineering, UIET, Punjab University, Chandigarh, 160014, India
}

\begin{abstract}
In this paper, behaviour of $\mathrm{A} 12 \mathrm{O} 3$ in aluminium matrix composites is reviewed for its properties and applications. In addition, many metal matrix composite fabrication processes are also elaborated. In the present days the aluminium metal matrix composite is in high demand because of its superior properties. Its demand is still on rise because of its widespread use in automotive industries, aerospace industries and marine industries. The method of the fabrication of aluminium matrix-based composite is also a deciding factor for its resultant properties. Desired composite-properties are achievable by proper selection of reinforcing materials as well as the physical conditions. Various sections of current information compile the details about the behaviour of alumina particles in aluminium-based matrix for formation of metal matrix composites.
\end{abstract}

\section{Introduction}

In last some decades light metal matrix composites (MMCs) materials having ceramic reinforcement particles have received widespread attention because of having superior mechanical properties over the base materials and found many interesting applications [1], [2]. Behaviour of alumina $\mathrm{Al} 2 \mathrm{O} 3$ particles present in the MMC play an important role in deciding the overall properties of such composites [3]. Among the several reinforcements, alumina $\mathrm{A} 12 \mathrm{O} 3$ particulate reinforced aluminium composites (Al-A12O3) are having superior mechanical properties so these are treated as one of the best successful MMCs available till date [4]. In MMCs, the aluminium and alumina have been progressively more useful in different sectors like in aircraft or automotive industries and aerospace industry because this $\mathrm{Al}-\mathrm{Al} 2 \mathrm{O} 3 \mathrm{MMC}$ have high strength-to-weight ratio, better tribological properties and good cast-ability over the base materials or unreinforced alloys [5]. When we manufacture or fabricate the $\mathrm{Al}-\mathrm{Al} 2 \mathrm{O} 3$ composite via the powder metallurgy (PM) process by taking mixture of aluminium and alumina powder usually we get weak bonding at the aluminium and alumina interface because of weak wettability between the two phases. In case of MMCs we get the desired mechanical properties like strength, hardness, fatigue resistance and wear resistance etc. MMC having aluminium as base material are called aluminium matrix composites (AMCs) [6]. In AMCs the reinforcements are particles and whiskers and AMCs are commonly useful for very critical and severe domain applications and components making, now a days these are very useful in the field of aviation, military, automobile sector, electricity, shipbuilding and aerospace sectors due to these AMCs having very good mechanical and physical properties in comparison of other materials [7].

In the present industrial requirement alumina-based MMCs are much important to meet the desired requirements. Al-A12O3 having reinforcement particles of alumina of nano size, these alumina-based matrix materials satisfy the present requirements of several industries and show the superior microstructural and physical properties that fulfil the present need of the industries. Because nano particles have very small dimensions so it is easy to attain more accuracy, nano particles impart the property to develop new features and the resulting composite can perform several new functions which are impossible even by large size / structure materials [8]. It is easy to change or alter the chemical and physical properties such as stability, reactivity, hardness, optical sensitivity, melting point, thermal and electric conductivity because of very small dimensions of nano particles [9].

Among different aluminium alloys, the reason behind the selection of aluminium-5083 alloys is its excellent strength with very light weight in comparison to other alloys and this alloy shows very good properties even at very low temperatures, very high toughness even at cryogenic temperatures that is very near to zero, its better weldability and good corrosion resistance. It is very commonly used in ship and its components building and cryogenic industries. Aluminium-5083 alloy also performs much better corrosion resistance to saline water and the marine environment and exhibits well enough mechanical properties and have high enough fatiguefracture resistance [6]. Aluminium-5083 alloy is used to manufacture components of railway, marine ships, boat

\footnotetext{
* Corresponding author: sehgals@pu.ac.in
} 
panels and platforms, train panels and platforms, thick and thin pressure vessels, oil storage tanks, cryogenics, tubes and pipes for oil industries, industrial trailers and tanks, automobile industries dump bodies, structures of automotive industries, railway and civil bridges, components of army vehicles and armour plates. It is also used for spacecraft and airplane bodies because of its light weight and well enough strength. Aluminium5083 alloy is not preferential for use above $650 \mathrm{C}$ for a continuous long time. If friction stir processing is done to aluminium-5083 alloy then this alloy shows superplastic properties with grain refinement [10].

\subsection{About MMC}

MMC is a composite material consisting of a minimum of two constituting parts, out of which one must be a metal in majority and the other being a different metal or even a non-metal (ceramic or organic compound) and the two must be non-soluble in each other. Presence of more than two constituting parts leads to the formation of what is called as a hybrid composite. In composites, superior properties as compared to the properties of the base materials are obtained which are not possible to be found separately [11]. We take a base material as the main compound or major phase; in this base material some foreign reinforcement particles are added which is generally named as reinforcement phase these are added for the formation of the composites. MMC are human made composites which are made by the combination of the base matrix and the hard reinforcement materials like alumina, silica carbide etc. Mixing of reinforcement is done to get desired physical or mechanical properties. MMC are used to manufacture components of railway, marine ships, boat panels and platforms, train panels and platforms, thick and thin pressure vessels, oil storage tanks, cryogenics, tubes and pipes for oil industries, industrial trailers and tanks, automobile industries dump bodies, commercial airliners, modern bicycles, structures of automotive industries, railway and civil bridges, components of army vehicles and armour plates. It is also used for spacecraft and airplanes bodies because of its light weight and well enough strength [3].

Generally, two or more than two components matrix and reinforcement are required for the formation of any MMCs. Generally, the one substance is taken as a metal or alloy, and the other substance or reinforcement particles are generally taken to be some ceramic. Our target in process of formation of MMC is to combine the two substances by either way. In some of the works aluminium MMCs (Al-MMCs) emerge as the boom for aerospace, automotive, shipbuilding and defence industries. Al-MMCs are light in weight, having high strength, very low thermal expansion coefficient and having very good wear resistance. In the current research framework, alumina $\left(\mathrm{Al}_{2} \mathrm{O}_{3}\right)$ is mostly added as reinforcement to aluminium matrix. Adding alumina $\left(\mathrm{Al}_{2} \mathrm{O}_{3}\right)$ reinforcement to aluminium matrix is a wellknown method of improving upon the mechanical properties. In the case of Al-MMC strength of the composite may be even 64 to $100 \%$ higher than that of the base aluminium matrix. Thermal stability of alumina is much better than that of common ceramic additives such as silicon carbide particularly under high temperature conditions. There exist so many methods for the fabrication of the reinforced aluminium matrix, but in all the methods ceramic particles/powders like silicon carbide ( $\mathrm{SiC})$, alumina ( $\mathrm{Al} 2 \mathrm{O} 3$ ), aluminium nitride (AlN) are generally added into the aluminium melt by any of the method like casting method or mixed with aluminium powder for further sintering; although the major difficulties arise in mixing alumina and aluminium together because alumina particles have low wettability and the resulting composites are highly anisotropic in nature because of the homogeneous distribution of the reinforcement particles.

Generally, when we mix the two or more material or phases of the same materials, the composite forms but the main condition for the materials is that the materials should be insoluble and should have the superior mechanical and metallurgical properties to the component materials which were taken for the mixing to form the composites. Nano-composites are much common type of the composites in present research scenario and defined as a class of materials in which at least one of the materials that we mix to other material should be of nanometer range $(1 \mathrm{~nm}=10-9 \mathrm{~m})$. The size, quantity and distribution pattern of reinforcement particles play a major role in limiting or enhancing all the mechanical and metallurgical properties of the MMC. When we mix smaller, submicron or nano sized reinforcement particles into the aluminium we get the desired composites of highly improved mechanical and metallurgical properties. The size of reinforcement decides the properties of the composite materials.

\subsection{Nanocomposites classification}

Nanocomposites can be classified as either ceramic, metal or polymer-based nanocomposites [7] as discussed next.

\subsubsection{Ceramic matrix nanocomposites (CMNC)}

In CMNC materials the ceramic particles occupy the main part of the volume that is a chemical compound from the groups like nitride, boride, oxides, silicides etc. In the maximum cases of this type of composites, a significant improvement is observed in electrical, optical, magnetic, tribological and corrosion-resistance etc. Major examples of $\mathrm{CMNC}$ based materials include $\mathrm{SiO} 2 / \mathrm{Ni}, \quad \mathrm{A} 12 \mathrm{O} 3 / \mathrm{SiO} 2, \quad \mathrm{~A} 12 \mathrm{O} 3 / \mathrm{TiO} 2, \mathrm{~A} 12 \mathrm{O} 3 / \mathrm{CNT}$, $\mathrm{A} 12 \mathrm{O} 3 / \mathrm{SiC}$ etc. [7].

\subsubsection{Metal matrix nanocomposites (MMNC)}

In MMNC some ductile metals / alloy matrix and some nano-sized reinforcements are combined to form the composite matrix. In MMNC, combined features of metal and ceramics can be achieved easily such as toughness and ductility with high modulus and high 
strength. Because of its desired properties MMNC are very good in the fabrication of high service temperature capabilities materials and high shear/compression strength. These composite materials have the potential to be used in applications, such as automobiles, aerospace industries etc. Some major MMNCs include $\mathrm{Al} / \mathrm{SiC}$, $\mathrm{Al} / \mathrm{Al}_{2} \mathrm{O}_{3}, \mathrm{Fe}-\mathrm{Cr} / \mathrm{Al}_{2} \mathrm{O}_{3}, \mathrm{Co} / \mathrm{Cr}, \mathrm{Fe} / \mathrm{MgO}, \mathrm{Al} / \mathrm{CNT}, \mathrm{Ni} /$ $\mathrm{Al}_{2} \mathrm{O}_{3}, \mathrm{Mg} / \mathrm{CNT}$ etc. [7].

In the present scenario, aluminium based MMNC possess excellent specific strength and weight properties best suited to be used as structural members in aerospace industry and automotive industry [2]. Because of the optimal combination of ductility and high strength aluminium based MMNC have many application areas. Comparing pure aluminium to, $2.0 \%$ volume nano- $\mathrm{Al}_{2} \mathrm{O}_{3}$ additions, the yield strength, hardness and tensile strength may improve by even up to $66 \%, 50 \%$ and $80 \%$ respectively.

\subsubsection{Polymer matrix nanocomposites (PMNC)}

Basically, PMNC are nano filled polymer composites. In a simple case, the adding of nanoparticulate to a polymer matrix can alter or enhance its performance and in some cases that enhancement may be to a superior level. On the basis of the nature of the nano-composites the properties of the nanoscale filler are described by the term nano filled polymer composites. Composites from this method yield as high-performance composites because well enough dispersion of the reinforcement materials in the base matrix occurred [7].

\section{Limitations Of Pure Aluminium}

Despite having good strength-to-weight ratio, aluminium is not as strong as stainless steel. Welding of pure aluminium is very difficult so some special techniques are required to weld the pure aluminium in industrial applications. It is abrasive to tooling, due to formation of the aluminium oxide coating that forms upon its surface. Corrodes easily, if suitable precautions not taken. Aluminium gets water-stained easily. Aluminium is difficult to paint and little expensive than steels. Aluminium is very light weight and also has low modulus of elasticity. Use of aluminium can badly affect the taste of the food, therefore it is not preferred over stainless steel in cooking applications. In pole industries, pure aluminium cannot be used for making the poles because of its low material strength and less flexibility near bolt circles. Last but not the least, aluminium is expensive compared to steel of the same strength.

\section{Benefits of Al2O3 mixing}

From the literature it is observed that when we increase the value of weight percentage of $\mathrm{Al}_{2} \mathrm{O}_{3}$ particles in aluminium based MMC then the value of hardness increases. Further when we add the alumina particles then tensile strength of such composites improved to a noticeable value. Moreover, $\mathrm{Al}_{2} \mathrm{O}_{3}$ composites possess higher density than the base matrix, which can be increased further by increasing the weight percentage of filler content. High density-based products have lesser vibration related issues during their structural dynamic design. The microstructural investigations have confirmed that the $\mathrm{Al}_{2} \mathrm{O}_{3}$ particles are uniformly distributed in the aluminium based MMC system. It is also well known that the aluminium based MMC have better wear resistance properties as compared to the base matrix counterpart.

\section{Major investigations of $\mathrm{Al} 2 \mathrm{O} 3$ in various aluminium grades}

Study of $\mathrm{A} 359 / \mathrm{Al}_{2} \mathrm{O}_{3}$ has been done by Kumar et al. [12] and influence of input parameters on tensile strength, hardness as well as microstructural properties was observed. They found that with an increase in weight percent of alumina particles, the value of hardness increases during casting and the highest value of hardness was attained when at the 8 wt. $\%$ of $\mathrm{Al}_{2} \mathrm{O}_{3}$ was maintained to be $8 \%$. [12]. Tensile strength also improved with increasing percent of alumina particles/reinforcement particles. The tensile strength of A359 was $103.7 \mathrm{~N} / \mathrm{mm} 2$ in non-reinforcement condition but when we add the reinforced particles to it its tensile strength increased to a maximum value of $148.7 \mathrm{~N} / \mathrm{mm} 2$ at $8 \%$ weight percentage of reinforcement particles. From the study of some researchers, it is also noticed that when we add the hard reinforcement like $\mathrm{Al}_{2} \mathrm{O}_{3}$ the elongation percentage of the composite material in the tensile test is also reduced because the value of brittleness increased on adding the alumina particles. It is also noticed that on addition of alumina particles tensile strength increased and because of this increment in tensile strength refinement of grains has been done and the grain size reduced and thus the number of grain boundaries increased in alumina casting composite during the solidification process. Beyond the $8 \%$ weight percentage of alumina and up to a value of $15 \%$ weight percentage it was experienced that tensile strength as well as ductility reduced in the cast condition because of the porosity in the stir casted aluminium matrix [12].

One another very successful technique for fabricating the aluminium MMCs is electromagnetic stir casting technique. Moisture or water content in reinforcement material/particles burn off by applying the heat source to the reinforcement particles initially. Heating is done because the moisture or water content can make porosity in composite materials or in MMC. In this way the chances of porosity get reduced in this method. The value of hardness of $\mathrm{MMC}$ is increased in direct proportion to the weight fraction of $\mathrm{Al}_{2} \mathrm{O}_{3}$. Tensile strength increased on the increment of weight percent of $\mathrm{Al}_{2} \mathrm{O}_{3}$. On the microstructural study it was found that smaller grain developed by electromagnetic stirring action and developed a well enough particulate matrix interface bonding.

\section{Experimental procedure: various fabrication methods}


Aluminium matrix composites (AMCs) can be manufactured with the help of various techniques of fabrication such as liquid-state (casting), solid state processes and liquid-solid processing.

\subsection{Liquid-state (casting) processes}

Examples of this liquid state process are squeeze casting, stir casting, vacuum infiltration, ultrasonic-assisted casting, pressure-less infiltration and dispersion method etc.

Squeeze casting technique, a type of liquid state casting process, is combination of two processes viz., casting and forging. In squeeze process metal is melted which later on gets solidified generally under effect of external pressure. First of all, the metal is preheated, then the metal melts, molten metal is then poured into a mold, then specific pressure is applied on the metal in liquid form and finally the reinforcement particles are mixed properly and then the final composite is taken out of die and punch system. This process has two types one is indirect and other is direct. For achieving the good quality of composite matrix some crucial process parameters are mold temperature, mold pressure and pressure duration, time delay in pressurizing the melt, time delay in pressurizing the melt, pouring temperature, die temperature and filling velocity [13]. Defect-free cast composites can be fabricated by the squeeze casting process by properly maintaining the process parameters, which in itself is a field that needs more investigations.

Stir casting process (vortex technique) is a type of liquid state casting process. This is generally useful for the manufacturing or fabrication of aluminium MMCs using the silicon carbide or alumina oxide as the reinforcement particles. In this process the reinforcements particles disperse very well into the molten metal or molten aluminium and can be casted in any desired shape of interest. Before the mixing of the reinforcement particles, it is important to make molten metal gas free to avoid the chemical reaction of the molten metal with atmospheric oxygen [14]. In order to ensure that the reinforcement particles are uniformly distributed in the metal, proper mixing is very much important. Therefore, to ensure that proper take place, a rotor is rotated inside the liquid metal that creates a vortex and the gas carrying the reinforcements is injected into the liquid. Fabrication can be shaped by conventional casting method [15]. In this process there may occur lot of issues like entrapment of gas, formation of slag in melt, because of these two issues melt can lead to some defects such as high porosity as well as micro defect. Unwanted reactions between the reinforcements and matrix can also take place. To avoid such problems, the process parameters such as melt holding temperature, melt stirring time, temperature of the molten metal, choice of suitable matrix and reinforcement should be adapted carefully [16].

In the ultrasonic assisted casting process ultrasonic wave of frequency range from $20 \mathrm{kHz}$ to $18 \mathrm{kHz}$ is used. In the frequency range of $20 \mathrm{kHz}$ to $18 \mathrm{kHz}$ mechanical vibration occurred. Some micro bubbles rise in the liquid in this process. After that explosion of these bubble occurs (this is called cavitation), the total energy developed in this process cannot be absorbed by the bubbles. The life span of bubbles is 6-10 second, in every cavitation cycle, explosion of bubbles take place with a 'hot spot' that hotspot attains a pressure of 1000 atm and a temperature of $5000^{\circ} \mathrm{C}$, and heating/cooling rates $>1,010 \mathrm{~K} / \mathrm{s}$ during microseconds transient.

In spray-Up process the reinforcement particles and matrix taken in the form of the chopped fibres and a hand operated spray gun is involved to spray matrix and reinforcement high pressure. Generally, in Spray-Up process roving glass is taken as the reinforcement that have to go past the spray gun where this is chopped with a gun. Independently at the same time the reinforcement and matrix can be sprayed by the spray gun. Process of curing can be performed at high temperature or even at room temperature. When this process is completed, mold is opened and material kept out from or ejection can be done. Type of composite fabricated decides the curing duration and it may vary according to composite fabricated. The mechanical properties of the parts or products fabricated by this process are not so good, this process is suitable for the fabrication of bulky product, rich in resin.

\subsection{Solid state processes}

There are lots of processes comes under solid state, for example, PM process with variations in the processing steps, i.e., using hot iso-static pressing, cold iso-static pressing, hot die pressing, using dynamic compaction [17].

PM technique is very common technique for easy fabrication of AMC. AMCs reinforced by carbides, borates, nitrides and oxides can be fabricated PM [18]. With the help of PM technique very complex or typical geometries or parts can be fabricated easily. This technique requires less temperature and is also costeffective. The technique also allows for mass production at very low cost. Proper steps of the PM process are powder mixing, then compacting it in a die and then sintering in a furnace. Some secondary operations can also be used later for achieving specific properties or dimensional precision. $\mathrm{PM}$ is the best process for the mixing of the reinforcement particles in the base matrix material, in this process very well mixing of reinforcement particles is done. Due to this well mixing of reinforcement in the base matrix, the mechanical properties of the fabricated composite are very good. In the PM process the factors that affect the process are amount of reinforcement, size of nano particle reinforcement, reinforcement surface type and reinforcement type and properties of composite fabricated [19]. Nano level composite fabrication is much complicated but on the other hand nano size composite consists very good mechanical and metallurgical properties.

Spark Plasma Sintering (SPS) process is field assisted sintering process and, in this manufacturing, /fabricated process low voltage, direct current, and 
pressure assisted sintering involved. This fabrication process is suitable for fabrication of composite product and parts of high density and very excellent grain size or grain growth [19]. This process follows the Joule heating principle and produce high heating rates. In this process at very small temperatures and in very small processing times it allows the development of high-density ceramics under uniform heating. Schematic diagram of Spark Plasma Sintering process shown below. In the present time spark plasma sintering process is much useful to develop lots of modern composite compounds. Hot pressing process and spark plasma sintering process are almost similar, manner of heat development and heat given to the desired material that is being sintered is the only main difference. In this process green compact that is in the form of powder is converted into parts or compounds, powder form green compact put in the graphite die and then that green compact (powder form) subjected to electric discharge developed by pulse electric discharge and external pressure. Study at the microscopic level shows that in SPS an electric discharge process happens because of that diffusion of material got accelerated. In this process applied outside pressure taken as $20-3000 \mathrm{~N} / \mathrm{m}^{2}$ and heating rate taken $100-1000^{\circ} \mathrm{C} /$ minute. Desirable behaviour of the parts and products can be achieved in this process because porosity can be controlled in this method. This SPS process is very fast so the probability of densification is very high and coarsening can be eliminated that generally occur in conventional process by this way mechanical properties can be enhanced. This process generally occurs in the environment of a shielded gas or empty space at atmospheric pressure. SPS process needs more investigations into its mechanism as well as future improvements.

Hot isostatic pressing (HIP) is based on combined PM and stir casting process. Mechanical operation of materials from which we want to make composite depends on amount of porosity, structure of material, dispersion which affect the mechanical behaviour of materials. In this process very high temperature and a uniform pressure is applied to the green compact (powder form) in all directions under the medium of argon gas that is taken in a closed thick vessel to make proper consolidation of the materials as shown in diagram shown in Figure below. Because Temperature and pressure are applied simultaneously it was named 'gas pressure bonding' [19]. Very dense and flawless ceramics can be synthesized by this hot isostatic pressing. In hot isostatic pressing the desired mechanical properties like ductility, creep and impact resistance are much improved. Hot isostatic pressing is verified as a best technique for improving mechanical properties for large number of materials till present date.

Microwave Sintering (MWS) makes use of the microwave energy. Electromagnetic spectrum covers the microwave as its integral section that travels with the wavelength range $1 \mathrm{~mm}$ to 1 meter, speed is equal to speed of light and similar to $300 \mathrm{GHz}$ to $300 \mathrm{MHz}$. Electromagnetic energy is converted into thermal energy in the process of microwave sintering. Microwave
Sintering process is novel, attractive, valuable, efficient and economical manufacture process.

\subsection{Liquid-solid processing}

Semisolid forming and compo-casting are examples of this processing mixing of two phases was not proper or thorough in the first and third group, determination of critical temperature for infiltration also very difficult and very harmful chemical reactions at the interface as well as problems due to wettability or fluidity at matrixreinforcement interface.

\section{Effect on mechanical and microstructural properties}

From the several researches it is observed that when we increase the value of alumina reinforcement then the value of hardness is increased significantly during casting stir process. It is also observed that when we increase the value of alumina reinforcement then the values of tensile strength of metal matrix is increased significantly during casting stir process. On increasing the sintering temperature and the amount of $\mathrm{Al}_{2} \mathrm{O}_{3}$ the tensile strength, hardness and compressive strengths increased [20]. One more thing that was found in this investigation is the tensile and compressive strength decreased sharply as the reinforcement and sintering reached the optimum value. When the value of reinforcement particles and the temperature of sintering are increased then value of mechanical properties like hardness, yield stress increased till reaching their optimum values and then there is sudden fall with further increase of alumina reinforcement and temperature. Aluminium alumina composite was fabricated by PM technique by Rahimian et al. [21]. The effects of particle size, sintering temperature and time were investigated and they reported that as we minimize the size of the particles and increase temperature and time of sintering the hardness increases and the best hardness of $76 \mathrm{HB}$ and strength of $318 \mathrm{MPa}$ was found with particle size of $3 \mu \mathrm{m}$ at sintering temperature of $600^{\circ} \mathrm{C}$ in 45 minutes. The impact of $\mathrm{Al}_{2} \mathrm{O}_{3}$ on $\mathrm{AMC}$ was also investigated by Canakci et al. [22] using the mechanical alloying process. In the mechanical alloying process $37 \mu \mathrm{m}$ size aluminium and $13 \mu \mathrm{m}$ size $\mathrm{Al}_{2} \mathrm{O}_{3}$ used and they showed as result that value of hardness increased on increasing the milling time and content of reinforcement and it is also found that increased amount of alumina affected both morphological properties and microstructural properties of aluminium matrix composite. Aluminium alumina AMC was fabricated with the help of PM process and liquid metallurgy by Raju [23] and investigation of different mechanical properties has been done and nano alumina particles effect on these mechanical properties of alumina matrix composites also observed. As the results it was observed that with the increasing of nano alumina particles hardness also increased and tensile strength also increased with alumina particles up to $1.5 \%$ of $\mathrm{Al}_{2} \mathrm{O}_{3}$ thereafter tensile strength will decrease. By using the different size of grit 
papers, samples of MMCs are prepared for microstructural examination. Then the proper etchant is used for the etching of samples, sometimes Keller reagent used. These etched samples then dried in air after that microstructure was taken by microscope [12].

\section{Conclusion}

Aluminium based MMCs having the nano scale reinforcement are very important and useful due to their specific applications in the area of aerospace, automobile, military, defence, sports equipment, medical science equipment, ship and its components building, electronic packaging, in engines, oil pipe industries. By using these nano composite materials, the life of the machine part and that of other equipment can be increased to a higher level. These composites are light weight and having very good mechanical and metallurgical properties. Aerospace fins and airline jet engines are required to be light weight and this is quite possible because of nano particles-based composites. These components need high strength and stiffness, erosion-resistance, low weight density. In automobile industries some parts such as that of brake systems need to be highly wear-resistant as well as good thermal conductors, these are now a days fabricated by the nano scale-based composites because of their excellent material properties.

\section{References}

1. D. K. Koli, G. Agnihotri, R. Purohit, Int. J. Latest Trends Eng. Technol. 2, 486 (2013)

2. M. Bragaglia, R. Montanari, G. Montesperelli, Corros. Eng. Sci. Technol. 54, 601 (2019)

3. B. V. Ramnath, C. Elanchezhian, R. M. Annamalai, S. Aravind, T. S. A. Atreya, V. Vignesh, C. Subramanian, Rev. Adv. Mater. Sci 38, 55 (2014)

4. B. Sadeghi, P. Cavaliere, A. Perrone, Part. Sci. Technol. 38, 7 (2020)

5. X. Qiu, L. Qi, J.-R. Tang, X.-Y. Cui, H. Du, J.-Q. Wang, T.-Y. Xiong, J. Mater. Sci. Technol. 44, 9 (2020)

6. A. Wakeel, M. S. Shahid, R. A. Pasha, Pak. J. Sci. 71, 266 (2019)

7. D. K. Koli, G. Agnihotri, R. Purohit, Procedia Mater. Sci. 6, 567 (2014)

8. A. M. Sadoun, M. M. Mohammed, A. Fathy, O. A. El-Kady, J. Mater. Res. Technol. 9, 5024 (2020)

9. W. Barakat, O. Elkady, A. Abuoqail, H. Yehya, A. EL-Nikhaily, J. Pet. Min. Eng. 22, 1 (2020)

10. A. C. Reddy, E. Zitoun, Int. J. Eng. Sci. Technol. 3, 6090 (2011)

11. A. S. Zayed, B. M. Kamel, T. Abdelsadek Osman, O. A. Elkady, S. Ali, Fullerenes, Nanotub. Carbon Nanostructures 27, 538 (2019)

12. A. Kumar, S. Lal, S. Kumar, J. Mater. Res. Technol. 2, $250(2013)$
13. I. A. Zhukov, A. A. Kozulin, A. P. Khrustalyov, N. I. Kahidze, M. G. Khmeleva, E. N. Moskvichev, D. V Lychagin, A. B. Vorozhtsov, Metals (Basel). 9, 1199 (2019)

14. C. Reddy, Int. J. Emerg. Technol. Appl. Eng. Technol. Sci. 4, 26 (2011)

15. S. Sivananthan, V. R. Reddy, C. S. J. Samuel, Mater. Today Proc. 21, 713 (2020)

16. M. S. N. Wani , M. S. Mhaske, Evaluation 6, (2019)

17. K. S. Alhawari, M. Z. Omar, M. J. Ghazali, M. S. Salleh, M. N. Mohammed, Procedia Eng. 68, 186 (2013)

18. A. C. Reddy, E. Zitoun, Int. J. Mater. Sci. 6, 147 (2011)

19. M. A. Ibrahim, Y. Sahin, A. Y. Gidado, M. T. Said, Glob. Sci. J. 7, 23 (2019)

20. S. S. Razavi-Tousi, R. Yazdani-Rad, S. A. Manafi, Mater. Sci. Eng. A 528, 1105 (2011)

21. M. Rahimian, N. Ehsani, N. Parvin, H. reza Baharvandi, J. Mater. Process. Technol. 209, 5387 (2009)

22. A. Canakci, T. Varol, S. Ertok, Sci. Eng. Compos. Mater. 19, 227 (2012)

23. K. Raju, A. Sathishkumar, S. Vignesh, D. Manikandan, Int. J. Adv. Eng. Recent Technol. 3, 35 (2015) 